\title{
Time Multiplexing-Star Shape Body Sensor Network for Sports Applications
}

\author{
Haider A. Sabti \& David V. Thiel \\ School of Engineering, Griffith University, Brisbane, QLD Australia \\ haider.al-husseinawi@griffithuni.edu.au, d.thiel@griffith.edu.au
}

\begin{abstract}
Health and sport applications have been a flourishing area for deploying Wireless Body Area Networks (WBAN) as this technology can provide a real time feedback which is important for the user, coaches, doctors and the viewing public. This paper presents the wireless channel reliability and efficiency for time multiplexing and star shape body area network operating on $2.45 \mathrm{GHz}$ with a data rate of $1 \mathrm{Mbps}$. The network employs sensor locations on both arms and legs of the human participant to sense and send acceleration data during the course of running. The results show a tradeoff between the channel occupancy and traffic generated to provide high channel reliability for the body network.
\end{abstract}

Keywords-WBAN; WPAN; BAN; Body Centric Network; Body Area Network; Time division; Sports; Running

\section{INTRODUCTION}

Recently, there has been increasing interest from researchers, system designers, and application developers on a new type of network architecture generally known as Body Sensor Networks (BSNs) or Wireless Body Area Networks (WBANs), made feasible by advances on lightweight, smallsize, ultra-low-power, and intelligent wearable sensors. A Body Area Network is formally defined by IEEE 802.15 as, "a communication standard optimized for low power devices and operation on, in or around the human body (but not limited to humans) to serve a variety of applications including medical, consumer electronics / personal entertainment and other" [1]. In more common terms, a Body Area Network is a system of devices in close proximity to a person's body that cooperate for the benefit of the user.

A WBAN consists of a collection of small sensor nodes placed around the body. These may be attached directly to the skin or as part of special clothing [2]. Each node has a small power source and the nodes collectively communicate with a central node (like a Personal computer or PDA) which can in turn connect to the internet or local host computer. The data from the sensors can be relayed to a particular application or person for monitoring and further processing. Utilizing WBAN in sports can provide a real time feedback for athletes which lead to a better understanding the players' performance and can help coaches to effectively measure training sessions. The provision of accurate information can prevent injuries and support the rehabilitation process through monitoring performance levels over time [3, 4]. The use of multiple access technologies provide consistent and burst signal transmission over time, frequency and code [5]; which can support the reliable communication for the wireless nodes without packet collision. This paper presents the use of a time division technique to allocate time segments for each on-body wireless node to transmit real time data during running to the wireless central node for further processing.

\section{SYSTEM MEASUREMENTS}

\section{A. Scenario}

The measurements have been carried out on an open sport field (free space) to minimize possible multipath effects from nearby objects. The network was designed to transmit packets at the same frequency channel $2.45 \mathrm{GHz}$ and data rate of $1 \mathrm{Mbps}$. The transmitters use different time slots to send their data to the receiver in periodic manner and consecutive sequence starting at transmitting node TX1 and ending at transmitting node TX4. The time difference between each transmission was calculated to allow for data transmissions within a sampling rate of $20 \mathrm{~Hz}$ (i.e. time between transmissions burst is $12.5 \mathrm{~ms}$ which is more than the time required to transmit and acknowledged the data packet). Each transmitter has a different address allowing the receiver to identify the origin of the packet.

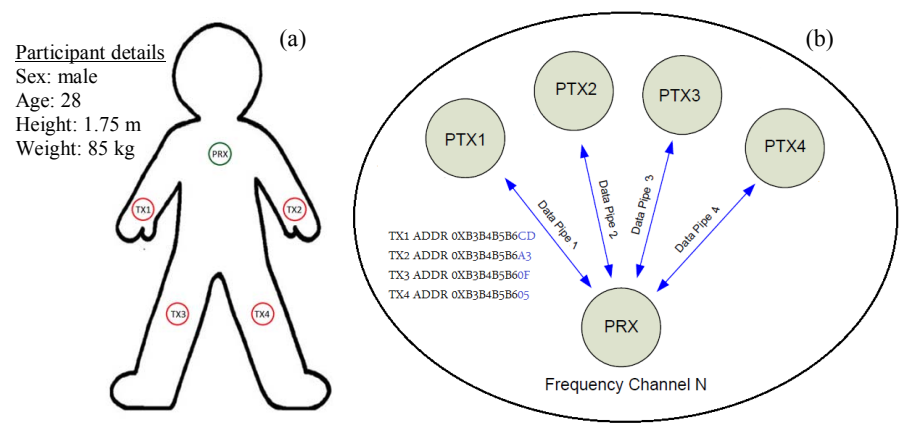

Fig. 1. The primary receiver node and transmitter nodes (a) location on the human body (b) address characteristics.

Fig.1 shows the star network topology used for the five wireless transceiver nodes, in which four transmitters are located on the arms and legs of the human's participant and one receiver (hub node) placed at the chest. For stability and easily on body mounting, the nodes were attached in fabric 
bands to outside part of the wrists and the thighs just above the knee. The primary receiver sensor node programmed to log only the data received from the transmitter nodes and to log nothing when there is no transmission (transmission stops and the data is lost). All wireless nodes have the same hardware structure but they were programmed with different code to perform their tasks in $\mathrm{C}$ language. Each node consisted of a MCU (ATmega324P Microcontroller), wireless transceiver chip module (NRF24L01+) with a built-in PCB trace antenna that allows multi-reception from up to six paths, accelerometer sensor (MMA7260QT), memory (AT45DB161D) and a power source in the form of $3.6 \mathrm{~V}$ battery with a maximum capacity of $240 \mathrm{uA}$ that can provide active node operation around three hours. This is sufficient to monitor of one complete training session. Each node has a UART interface (CP2102) which allows wired data transfers to a local computer for real-time monitoring or further processing during or after the completion of the sport activity.

\section{B. Measurement Results}

This paper follows previous work on the analysis of the wireless link performance in body area networks [6], and reports a real time feedback star shape body sensor network using movement based time division multiplexing. The network deploys the hub node to collect sensed data from each transmitting node during running. Each node sends the sensed acceleration data every $50 \mathrm{~ms}$, and accordingly the hub node receives four transmissions each $50 \mathrm{~ms}$ (12.5ms apart).

The auto acknowledgement feature of the wireless transceiver chip (NRF24L01+) was activated in this network. This feature enables the transmitter to wait for the receiver acknowledgement to know whether the packets were successfully received. After data transmission the auto acknowledgement switches the transmitter to receive mode and to wait for the ACK packet. If the transmitter does not receive the ACK packet, the original data packet is retransmitted after a programmable delay of $(250 \mu \mathrm{s})$ and the process continues for up to 15 retransmissions until successful delivery or the maximum number of retransmissions was reached.

TABLE I

Wireless link evaluation for each wireless transmitter node

\begin{tabular}{cccc}
\hline \hline Node & Total traffic kbps & Throughput kbps & Reliability \\
\hline TX1 & 23.81 & 1.55 & $97 \%$ \\
TX2 & 23.79 & 1.53 & $96 \%$ \\
TX3 & 19.89 & 1.51 & $94 \%$ \\
TX4 & 23.73 & 1.49 & $93 \%$ \\
\hline \hline
\end{tabular}

Table I shows the total traffic generated, the throughput and link reliability for each transmitter node. From the table it can be seen that the generated traffic is much bigger than the node throughput. This traffic was produced from the retransmission attempts of each transmitter and resulted in a high aggregated body network reliability of $95 \%$. Nevertheless it reduces the link capacity and restricts the number of transmissions, nodes and information size in large networks. Thus link capacity is lower when the link reliability is important. The channel efficiency is defined by the utilization of wireless transmission channel with respect to its data rate. In this scenario, knowing that each data packet is 80bits long and the sampling rate is $20 \mathrm{~Hz}$ per transmitter with $1 \mathrm{Mbps}$ data rate, the network total channel efficiency is $9 \%$ (that means the channel is free for $91 \%$ of the time). Therefore, the large amount of traffic generated would only affect the network life time as more transmissions will occur in favor of robustness.

\section{ACKNOWLEDGEMENT}

This work was supported by the Higher Committee for Education Development in Iraq (HCED). This research was conducted under Griffith University Ethics approval number ENG/20/13/HREC.

\section{CONCLUSION}

A body sensor network utilizes time division multiplexing for capturing real time gait running movement. High aggregated network reliability of $95 \%$ was achieved by retransmitting the lost packets to increase the chance of information delivery. The drawback is the network life time will become shorter as it will consume more power compared to its normal operation. Future work will include the optimization of the central node functions to provide both on and off body communications and the ability to include multihop communication between the hub nodes of different players to increase system reliability and assist in information delivery to its required destination.

\section{REFERENCES}

[1] (2013, 22th November 2013). IEEE 802.15 WPAN Task Group 6 (TG6) Body Area Networks

[2] B. Latré, B. Braem, I. Moerman, C. Blondia, and P. Demeester, "A survey on wireless body area networks," Wirel. Netw., vol. 17, pp. 1-18, 2011.

[3] D. Brunelli, E. Farella, L. Rocchi, M. Dozza, L. Chiari, and L. Benini, "Bio-feedback system for rehabilitation based on a wireless body area network," in Pervasive Computing and Communications Workshops, 2006. PerCom Workshops 2006. Fourth Annual IEEE International Conference on, 2006, pp. 5 pp. -531.

[4] J. Neville, A. Wixted, D. Rowlands, and D. James, "Accelerometers: An underutilized resource in sports monitoring," in Intelligent Sensors, Sensor Networks and Information Processing (ISSNIP), 2010 Sixth International Conference on, 2010, pp. 287-290.

[5] C. Hsiao-Hwa and M. Guizani, "Multiple access technologies for B3G wireless communications," Communications Magazine, IEEE, vol. 43, pp. 65-67, 2005.

[6] H. A. Sabti and D. V. Thiel, "Overall performance of a wireless body area network in sports and running applications," IEEE Sensors Journal 2014. [under review] 